\title{
Tackling Dialysis Burden around the World: A Global Challenge
}

\author{
Philip Kam-Tao Li ${ }^{a}$ Gordon Chun-Kau Chan ${ }^{a}$ Jianghua Chen ${ }^{b}$ \\ Hung-Chun Chen ${ }^{c}$ Yuk-Lun Cheng ${ }^{d}$ Stanley L.-S. Fan ${ }^{e}$ John Cijiang He ${ }^{f}$ Weixin Hu ${ }^{g}$ \\ Wai-Hon Lim ${ }^{\mathrm{h}}$ York Pei ${ }^{i}$ Boon Wee Teo ${ }^{j}$ Ping Zhang ${ }^{\text {b }}$ Xueqing Yu ${ }^{k}$ Zhi-Hong Liu ${ }^{g}$ \\ aDepartment of Medicine and Therapeutics, Carol and Richard Yu Peritoneal Dialysis Research Centre, Prince of \\ Wales Hospital, The Chinese University of Hong Kong, Shatin, Hong Kong SAR; bidney Disease Center, the First \\ Affiliated Hospital, College of Medicine, Zhejiang University, Hangzhou, China; ' Department of Internal Medicine, \\ Kaohsiung Medical University Hospital, Kaohsiung, Taiwan; ${ }^{d}$ Department of Medicine, Alice Ho Miu Ling Nethersole \\ Hospital, Tai Po, Hong Kong SAR; 'Department of Renal Medicine and Transplantation, Barts Health NHS Trust, \\ London, UK; 'Division of Nephrology, Department of Medicine, Icahn School of Medicine at Mount Sinai, \\ New York, NY, USA; 9 National Clinical Research Center of Kidney Diseases, Jinling Hospital, Nanjing University \\ School of Medicine, Nanjing, China; hepartment of Renal Medicine, Sir Charles Gairdner Hospital, Perth, WA, \\ Australia; 'Department of Medicine, University of Toronto and University Health Network, Toronto, ON, Canada; \\ jDivision of Nephrology, Department of Medicine, Yong Loo Lin School of Medicine, National University of \\ Singapore, Singapore, Singapore; 'Department of Nephrology, Guangdong Provincial People's Hospital \& \\ Guangdong Academy of Medical Sciences, Guangzhou, China
}

\section{Keywords}

Chronic kidney disease · End-stage kidney disease .

Prevention · Home dialysis · Kidney transplantation

\begin{abstract}
CKD is a global problem that causes significant burden to the healthcare system and the economy in addition to its impact on morbidity and mortality of patients. Around the world, in both developing and developed economies, the nephrologists and governments face the challenges of the need to provide a quality and cost-effective kidney replacement therapy for CKD patients when their kidneys fail. In December 2019, the 3rd International Congress of Chinese Nephrologists was held in Nanjing, China, and in the meeting, a symposium and roundtable discussion on how to deal with this CKD burden was held with opinion leaders from countries and regions around the world, including Australia, Canada, China, Hong Kong, Singapore, Taiwan, the UK, and the USA.
\end{abstract}

The participants concluded that an integrated approach with early detection of CKD, prompt treatment to slow down progression, promotion of home-based dialysis therapy like peritoneal dialysis and home HD, together with promotion of kidney transplantation, are possible effective ways to combat this ongoing worldwide challenge.

$$
\begin{aligned}
& \text { (c) } 2021 \text { The Author(s) } \\
& \text { Published by S. Karger AG, Basel }
\end{aligned}
$$

\section{Introduction}

CKD is a global problem that causes significant burden to the healthcare system and the economy. Around 850 million people are currently suffering from different types of kidney disorders, while one in ten adults worldwide has

Philip Kam-Tao Li is the key corresponding author and Zhihong Liu is the co-corresponding author.

\section{karger@karger.com www.karger.com/kdd

\section{(C) 2021 The Author(s)}

Published by S. Karger AG, Basel

This is an Open Access article licensed under the Creative Common Attribution-NonCommercial-4.0 International License (CC BY-NC) (http://www.karger.com/Services/OpenAccessLicense), applicable to the online version of the article only. Usage and distribution for commercial purposes requires written permission.
Correspondence to:

Philip Kam-Tao Li, philipli@ cuhk.edu.hk

Zhi-Hong Liu, liuzhihong@ nju.edu.cn 
CKD $[1,2]$. CKD is projected to become the 5th most common cause of years of life lost globally by 2040 [3]. Prevention, early detection, and prompt treatment are cost-effective measures to prevent subsequent progression of CKD and development of end-stage kidney disease (ESKD). In order to raise public awareness of the kidney disease burden and promote early detection and treatment, the theme of World Kidney Day 2020, a joint initiative of the International Society of Nephrology and the International Federation of Kidney Foundations, was "Kidney Health for Everyone Everywhere - from Prevention to Detection and Equitable Access to Care" [2]. Renal transplantation is a highly effective cure of ESKD, but it is limited by the scarce supply of donor kidneys. For patients without a donor kidney, renal replacement therapy using dialysis is the best option to treat ESKD and to prolong survival. By 2010, 2.6 million people worldwide received renal replacement therapy [4]. However, initiation and maintenance of dialysis requires high burden of resources; for example, a dedicated team of well-trained healthcare professionals, special dialysis equipment, and facilities. These may not be readily available in certain part of the world. It is estimated that at least 2.3 million people might have died prematurely because RRT could not be accessed, in particular in low-income countries like Asia and Africa [4]. A report has shown that dialysis therapy consumes $2-3 \%$ of the annual healthcare budget in high-income countries [5]. In order to address the current CKD burden and to discuss plans in different parts of the world to make managing ESKD sustainable, a symposium and roundtable discussion was held on 6 December 2019 in Nanjing during the 3rd International Congress of Chinese Nephrologists. Opinion leaders from different countries and regions around the world, including Australia, Canada, China, Hong Kong, Singapore, Taiwan, the UK, and the USA presented current local data and potential solutions being explored in their countries.

\section{Australia}

In Australia, the sex- and age-specific incidence rates of patients with treated CKD are comparable to those in European countries but are substantially lower than those in other countries like the USA, Canada, and many Asian countries [6]. Up to $35 \%$ of the ESKD cases were directly related to diabetes mellitus. Although CKD patients' survival rates have improved in the past decades, these survival rates are still substantially lower than those in the general population and also in patients affected by certain cancers including colorectal and breast cancers [7]. In
$2014,22,218$ patients died of kidney-related diseases, and it ranks as the fifth commonest cause of death in Australia. Late referral, defined as the initiation of dialysis within 90 days of referral to renal service, is one of the major contributors of premature mortality among kidney failure patients [8]. To improve this situation, the Australian government implemented a number of policies to enhance early recognition of CKD, including the automatic laboratory reporting of the estimated glomerular filtration rate, the use of Kidney Health Australia's promotion of kidney health checks, which includes the assessment of blood pressure, glomerular filtration rate, and albuminuria in high-risk patients, as well as the implementation of the comprehensive, multipronged primary healthcare CKD education programs by the Primary Healthcare Education Advisory board for Kidney Health Australia's (PEAK) [6]. As a result, there was a drastic 32\% reduction in late referrals for treated ESKD in past decades, with the latest reported rate of $18 \%$ in 2017 [9].

CKD also imposes a substantial burden to the healthcare and economic systems. Patients with CKD incited $85 \%$ higher healthcare costs and 50\% higher government subsidies than individuals without CKD. Dialysis and related treatments also accounted for $13 \%$ of overall hospitalizations in Australia, with a projected average increase of 3.6\% each year [7]. To reduce the financial burden, kidney transplantation and home-based dialysis treatment are often promoted over hospital-based dialysis therapy in appropriate patients. Home-based dialysis is less costly as it requires lower infrastructure and staffing ratios than hospital or satellite dialysis centers. The KHA economic health report 2010 estimated that up to AUD 4 billion can be saved with a greater uptake of home treatment dialysis options (home HD and peritoneal dialysis $[\mathrm{PD}])$ than hospital-based treatment for patients with kidney failure, with even more cost savings achievable through increased kidney transplantation rates. Given the low proportion of Indigenous patients being transplanted, a greater focus on promoting kidney transplant access for Indigenous patients with kidney failure, especially those residing in very remote areas, is being deliberated.

\section{Possible Ways to Address the Burden}

- Clinical education and preventive programs need to be augmented to promote $\mathrm{CKD}$ recognition and close monitoring of progression in individuals at risk of CKD or with established CKD (to counteract lack of 


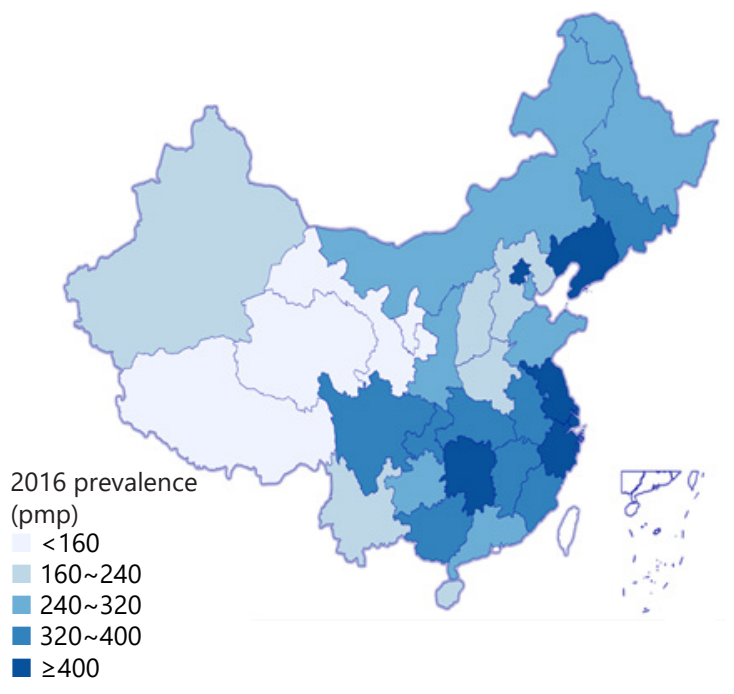

PMP of dialysis in china (2016)

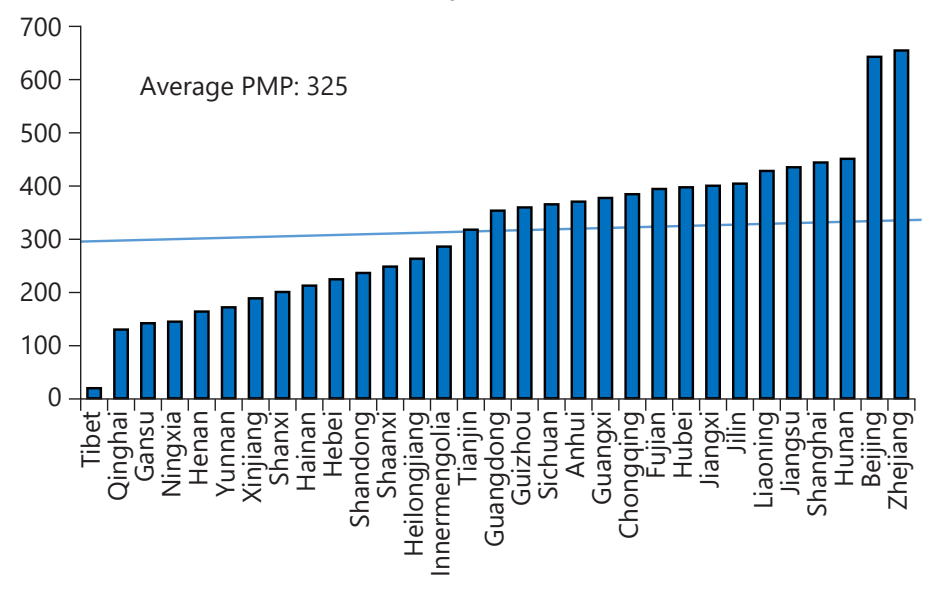

Fig. 1. Regional variation of prevalence of dialysis patients in different provinces showing the uneven distribution in China (Courtesy of Chinese National Renal Data System). pmp, per million population.

awareness and low detection rates of people with CKD among primary healthcare physicians);

- concerted, nationwide effort to implement integrated chronic disease management strategies, including improved coordination and communication regarding patients with CKD between primary and specialist care physicians to ensure timely access to appropriate clinical investigations and treatment;

- patient autonomy and appropriate support in the choice of RRT, particularly home dialysis treatments such as PD and home hemodialysis, should be provided by all renal centers while ensuring timely access to transplantation; and

- the need to establish CKD registries or data linkages to other health information systems in order to capture the true rates of CKD and untreated ESKD, to complement the data collected in the Australia and New Zealand Dialysis and Transplant (ANZDATA) registry, and to better inform future healthcare planning and resource allocation regarding the growing burden of CKD and ESKD in Australia

\section{Canada}

Over the past 2 decades, the incident number of ESKD patients older than 65 years in Canada surged, while that of patients younger than 65 years remained

Tackling Dialysis Burden around the World similar [10]. In 2017, there were approximately 38,800 patients with ESKD, which comprised around $0.14 \%$ of the population. 5,559 (14\%) of them were incident patients. Diabetes mellitus is the commonest cause of kidney failure, which accounted for 39\% of all the ESKD patients. Among patients with ESKD, $60 \%$ are on dialysis treatment and majority of them use HD (75\% HD, $25 \% \mathrm{PD})$. The other $40 \%$ of the ESKD patients were treated with a kidney transplant (60\% deceased vs. $40 \%$ living donor).

In Canada, PD patients have a better survival than HD patients before adjusting for comorbidities (5-year survival rate: PD 55\% vs. HD 43\%). Dialysis patients' survival also depends on age as the survival rate decreased in a stepwise manner, from $77.8 \%$ in age $18-44$ years to $26.8 \%$ in age greater than 75 years, regardless of their dialysis modality. Among the primary causes of kidney disease, polycystic kidney disease followed by glomerulonephritis conferred the best survival rate, whereas diabetes, renal vascular, and drug-related kidney disease conferred the worst survival among dialysis patients [11].

There is a significant increase in deceased kidney donations from 541 in 2012 to 803 in 2017, while living kidney donations remained similar of 538 in 2012 to 535 in $2017[10,11]$. Such an increase in cadaveric transplantation helps in the relief in dialysis burden for patients with a better quality of life for the ESKD patients. 


\section{China}

CKD is an important health problem in China. The number of dialysis patients doubled from 2012 to 2017. At the same time, China represents the biggest population with diabetes mellitus and in the year 2013, there were 98.4 million diabetic patients aged 20-79 years in China [12]. Therefore, it is not surprising that the ESKD caused by diabetes mellitus is increasing rapidly in the country. In 2017, there were 524,467 prevalent HD patients and 86,344 prevalent PD patients in China [13]. In 2017, there were 90,166 newly initiated HD patients and 15,057 newly initiated PD patients in China, with a ratio of 86 versus $14 \%$ for HD versus PD incident patients, respectively [13]. There were 5,479 HD centers and 983 PD centers in 2017. There is a regional variation in the dialysis prevalence rate in China, ranging from 0.03 to $0.39 \%$, with the lowest prevalence rate at northwest China and the highest prevalence rate at central China (Fig. 1). The overall prevalence rate for dialysis was 325 per million population ( $\mathrm{pmp}$ ).

In $2015,4.8 \%$ of the overall hospitalization in the country was accounted by CKD and associated diseases [14]. The total medical expenditure of in-patients with CKD was more than USD 3 billion, which was $6.34 \%$ of the overall costs [14]. The median annual overall cost per patient was lower among PD patients than HD patients.

In 2009, the Chinese government launched a national healthcare reform [15]. The new system provides 2 major types of medical insurance to the citizens - the New Cooperative Rural Medical Scheme (NCRMS) and the Urban Basic Medical Insurance (UBMI). The former is mainly for all rural residents, while the latter is for urban employees, retired citizens, nonemployed residents, students, and children [16]. The total medical expenditure for dialysis patients was 429 million RMB in 2015, of which $76.61 \%$ was covered by the UBMI. Overall, the number of HD and PD patients constituted only 0.16 and $0.02 \%$ of individuals covered by the UBMI, while they consumed 2.08 and $0.34 \%$ of the overall UBMI expenditure.

In order to support the ESKD patients in rural areas and to improve the quality of dialysis service, the National Dialysis Training Program in county hospitals, sponsored by the Ministry of Health (MOH) and Chinese Nephrologists Association, was undertaken in 2013-2015. It involved 150 training centers in university hospitals and 800 recipient centers from 29 provinces over China. Up to 6 months, free training was provided to doctors and nurses. The training and recipient centers were evaluated and accredited by the $\mathrm{MOH}$ to ensure service quality.
The study-reported dialysis population that is under NCRMS medical care had higher all-cause mortality than patients under the UBMI [17]. This observation can be attributed to their lower socioeconomic state, lack of awareness, and the difficulty to access dialysis service and facilities as most of them live in rural areas.

Healthy China 2030 was released by the Chinese government on October 2016 for ensuring that the Chinese population has access to health, through advocating the whole society's participation in the concept of "Health for All, and All for Health." The implementation of equalization of basic public health services provides urban and rural residents with guaranteed access to the most effective basic health services. The public health service system will be adjusted and improved so that ordinary residents will have their health problems diagnosed earlier and so get earlier treatment. The plan also aims to further ease people's financial burden when paying for health and medical treatment by reducing the percentage of overall health expenditure paid by individuals and to meet an increasing variety of health requirements [18].

\section{Hong Kong}

In Hong Kong in 2018, there were 172 incident ESKD patients pmp annually, which ranked the 17th highest number of ESKD patients pmp in the world [19]. Like other parts of the world, diabetes mellitus contributes to the majority cause of kidney failure [20]. In Hong Kong, majority (90-95\%) of the dialysis patients are managed in the public sector under the government-funded Hospital Authority. Since the initiation of dialysis service in the 1980s, Hospital Authority adopted a "PD first" policy, that is, all CKD patients who require dialysis will be started on PD under public healthcare coverage unless contraindicated. As a result, up to $75 \%$ of dialysis patients are on PD, which made Hong Kong the city with the largest PDto-HD patient ratio in the world. PD offers several advantages over HD, including a lower cost, a higher effectiveness of quality-adjusted life years, and less dialysis - associated infection and a better preservation of residual renal function [21-23]. PD patients in Hong Kong have a comparable survival rate to HD patients even in the elderly group $[24,25]$.

Apart from a high PD-to-HD ratio, Hong Kong also ranked the second highest percentage (11.2\%) of home HD patients among prevalent HD patients, only after New Zealand (24.3\%) in 2016 [26]. Local data reported that home HD confers a better patient outcome with a 
significantly higher survival than hospital HD patients, the costs of initiation and maintenance of home HD, in both societal and healthcare providers' perspectives, are much lower than those of hospital HD [22]. Home HD should be considered a dialysis choice to those who have PD failure or contraindications to PD.

Thus, home therapy's first policy is promoted in the Hong Kong setting in order to tackle the dialysis burden, especially with a better cost-effectiveness and quality of life for patients, especially in the era of tightness of manpower supply of healthcare workers and reduction of risk of infection under pandemics like COVID-19 [21].

\section{Singapore}

Incident dialysis patients increased constantly, from 977 per year in 2013 to 1,175 per year in 2017 [27]. More older patients entered dialysis programs, increasing the median age of incident dialysis patients from 61 years in 2008 to 64.5 years in 2017. Men outnumbered women in both incidence and prevalence rates of dialysis, and the ethnic Malays had the highest incidence and prevalence rates of dialysis. Like other countries, diabetic mellitus is the major cause of CKD (>55\%).

The Singapore government introduced public health programs to combat the combined problem of the aging population, declining labor force, and increasing CKD prevalence with growing economic burden, and to maintain the sustainability of the healthcare system. One example is the Holistic Approach to Lowering and Tracking CKD (HALT-CKD) program established by the $\mathrm{MOH}$ [28]. It targets patients who receive subsidized public healthcare in government-run primary care polyclinics. This program helps patients control CKD risk factors by early identification and close tracking of CKD and disease control. By 2019, more than 49,000 patients joined the program. Over $90 \%$ of them were put on renoprotective medications like renin-angiotensin system blockers. More than one-third of them with poorly controlled diabetes achieved their blood glucose targets within one year of joining the program. The patients identified with CKD stage G4 are referred to nephrology services for further assessment and counseling on risks of ESKD. By earlier identification of advanced CKD, patients receive recommended ESKD treatment. This will avoid expensive complications and unnecessary hospitalizations in the first year after diagnosis of ESKD [29].

In Singapore, HD is the most prevalent dialysis modality, accounting for $87 \%$ of all dialysis treatments. And,

Tackling Dialysis Burden around the World
$98.6 \%$ of them had thrice weekly HD [27]. Most of the HD treatments in Singapore are performed in voluntary welfare organizations and private dialysis centers, whereas PD care and education are mainly provided by public acute hospitals. Assuming only chronic dialysis treatment costs and no doctor visits, hospitalizations, ancillary health visits, and medication costs, HD costs about 3,000 Singaporean dollar (SGD) per month, while PD costs 2000 SGD per month [30].

There are several funding sources available for medical care. They include MediShield Life, Medisave, and MediFund [31]. In essence, MediShield is a mandatory national insurance scheme which provides up to 1,000 SGD per month for dialysis; Medisave is a personal health saving account which pays for insurance up to an annual cap and allows payments for dialysis of 450 SGD per month; and MediFund only covers essential medical expenditure, and it does not cover dialysis cost [30]. In addition, patients who purchase private medical insurance integrated with MediShield Life enjoy increased dialysis benefits to the point where co-pays may be reduced to less than $5 \%$ of dialysis treatment costs. Aside from insurance coverage, several subsidies from charitable organizations including the National Kidney Foundation and Kidney Dialysis Foundation are available, in which the subsidy amount depends on patients' citizenship and permanent residency status, and per capita household income. Despite the multiple options for financial support on medical expenditure, there are still several gaps in funding and services; these include the lack of support for transportation and caregivers and an inadequate framework for palliative dialysis and conservative kidney care.

\section{Taiwan}

In 2016, Taiwan has the highest incidence (493 ppm) and prevalence $(3,392 \mathrm{ppm})$ of ESKD in the world [26]. Diabetic mellitus is again the commonest cause of kidney failure, accounting for $46.1 \%$ of incident ESKD patients in the country. Majority of dialysis patients received HD (91.8\% HD, 7.7\% PD). The 5-year survival rate of dialysis patients is higher than that in America and Europe. According to the registry data from the Bureau of National Health Insurance, the incidence and prevalence of ESKD patients older than 55 years increased in the past 20 years, especially in the advanced age-group (older than 75 years). The increased dialysis burden in Taiwan may be related to the national insurance policy, increasing life expectancy of the general 


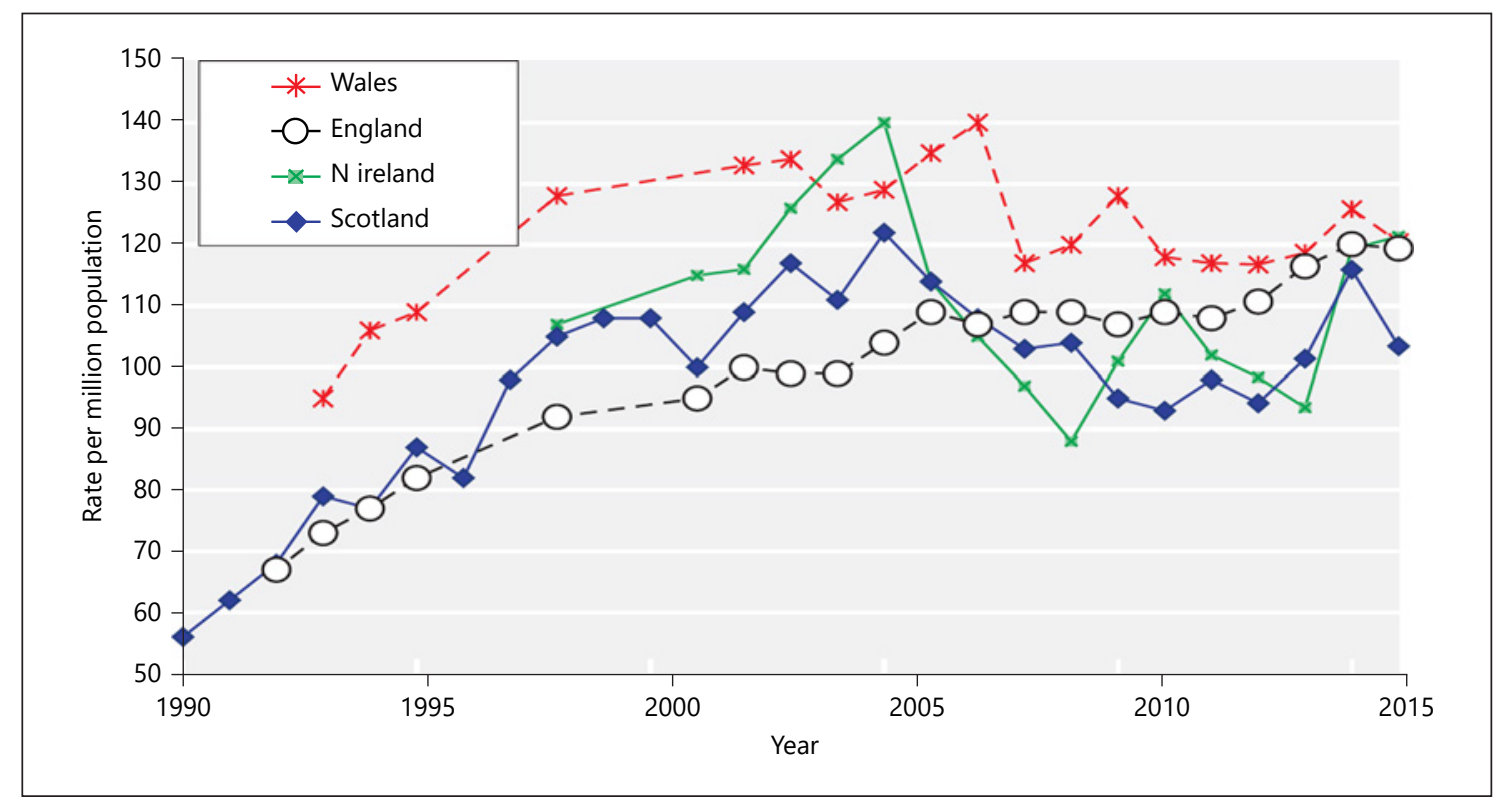

Fig. 2. Incidence rate of RRT in England, Scotland, Wales, and northern Ireland of the UK showing a plateau from 2010 to 2016. (The data reported here have been supplied by the UKRR of the Renal Association. The interpretation and reporting of these data are the responsibility of the authors and in no way should be seen as an official policy or interpretation of the UKRR or the Renal Association.) pmp, per million population.

population, better care of diabetics, low transplantation rate, possible nephrotoxic medications, and good survival of dialysis patients.

Because of the significant growth of prevalent dialysis population in Taiwan, the government tried to control the overall expenditure. In 2016, the reimbursement per HD treatment inclusive for ESA and all medications, and laboratory investigations was USD 105. The same figure for 2006 was USD 131. For PD, in 2016, the reimbursement per month was USD 1,433, and the same figure for 2006 was USD 1,423. The government was trying to give some incentives for PD usage.

To improve the care of CKD and retard the rate of CKD progression, the government started the pre-ESKD Care Project in 2007, which involved a wide range of strategies including urinary screening for high-risk populations; integrated care for stage $3 \mathrm{~B}, 4$, and $5 \mathrm{CKD}$ patients; and bonus payments for pre-ESKD care from insurance. This has resulted in a reduced all-cause mortality, death related to cardiovascular disease, infectious disease, cancer, and out-of-hospital cardiac arrest. The cost of initiating and maintaining dialysis was also significantly reduced [32]. The study concluded that the pay-for-performance program improved quality of predialysis CKD care and provided survival benefit and a long-term cost saving for dialysis patients [32].

\section{The United Kingdom}

The incidence rate of RRT has plateaued over the past years (Fig. 2). At the same time, there was a substantial growth of the in the elderly on dialysis in recent years. In order to tackle the dialysis burden, the following strategies have been employed: improve the pre-emptive live donor transplant rate, increase cadaveric transplant rates (opt out the system recently adopted in England), reduce late presenters starting of RRT, and increase PD utilization and supportive care for elderly comorbid frail patients.

In the UK, patients starting dialysis show a preference toward HD over the home-based dialysis therapy and PD. The RRT ratio for day 90 treatment of HD:PD:transplant has been roughly $70 \%: 20 \%: 10 \%$ from 2010 to 2016 . For late presenters ( $<90$ day to nephrologists), $12 \%$ start on $P D$, while with planned dialysis ( $\geq 90$ day to nephrologists), 22\% start on PD.

To ensure early detection of CKD with enhancement of the process of nephrologists' referral and to avoid emergency dialysis, the National Health Service (NHS) started a "virtual CKD clinics" (VC) project. Laboratory results from patients under general practitioner care were automatically uploaded to the Electronic Patient Records managed by the NHS. The patients were then immedi- 


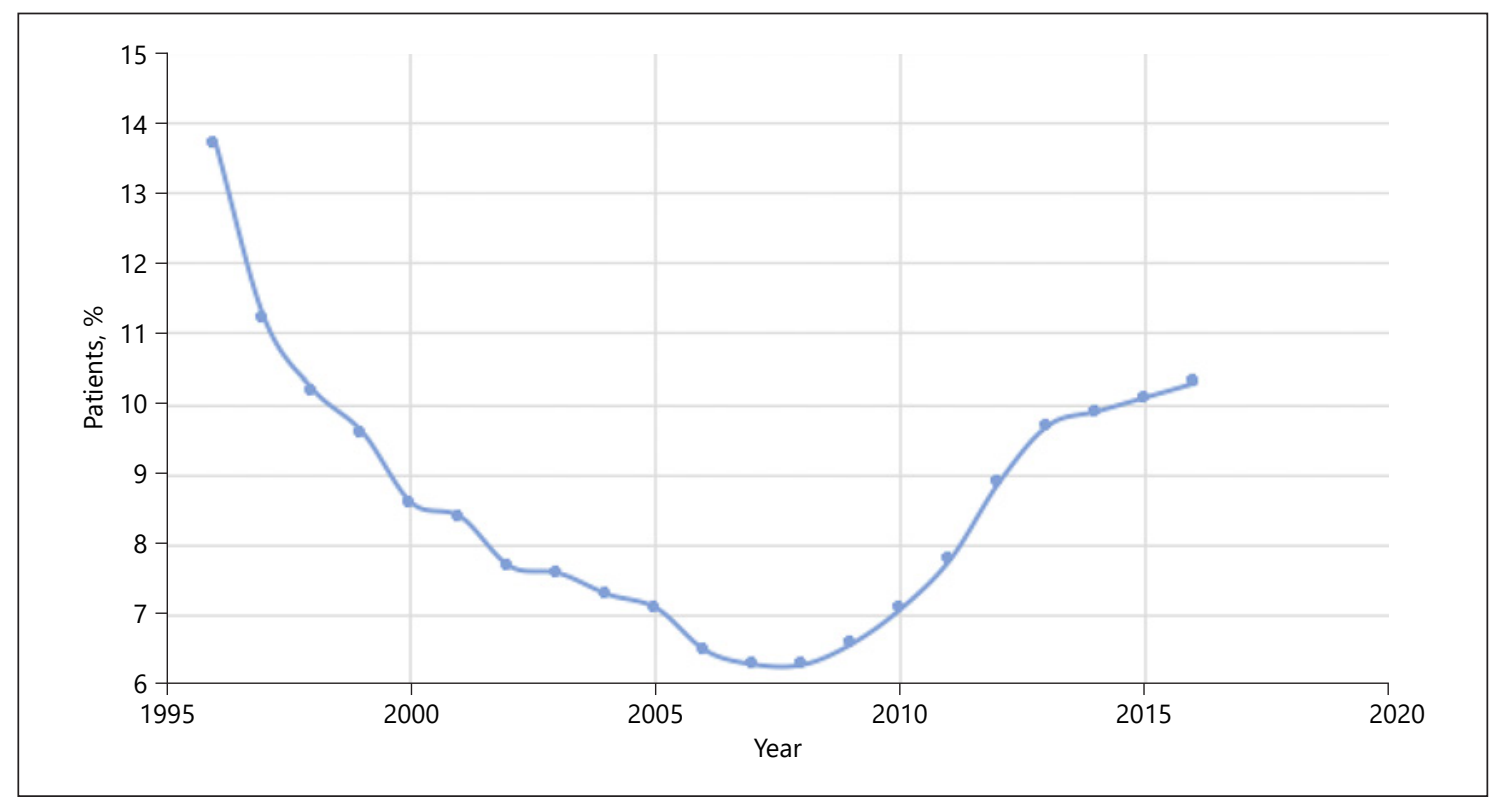

Fig. 3. Changes in the rate of patients undergoing home dialysis treatment in the USA from 1995 to 2016 (data source: Reference Table D.1 and special analysis, USRDS ESKD Database). ESKD, end-stage kidney disease.

ately referred to nephrologists when the computer system detects any deterioration in kidney function. This strategy not only recognizes disease early and permits early treatment but also provides sufficient time for the patients to understand the nature of disease and different modalities of RRT and make their decision before their kidney fails. Harnett et al. [33] reported that under this project, none of the patients from the $\mathrm{VC}$ required emergency dialysis, suggesting robust surveillance. Survival was similar to patients with CKD discharged to primary care.

A Primary Secondary Care Partnership to Improve Outcomes in Chronic Kidney Disease study was started in the UK. In 23 intervention practices (11,651 patients), a CKD nurse practitioner worked with nominated practice leads to interpret the data file and implement guideline-based patient-level CKD management interventions. With the significant improvement in the overall care process, the burden and associated costs of CKD and associated disease including cardiovascular disease burden reduced significantly [34].

\section{The United States}

The total expenditure on ESKD has consistently increased since 2004. In the USA, majority of the expenditure is covered by Medicare Fee-For Service paid, fol- lowed by Medicare Managed Care and Patient Care. In 2015, the Medicare system spent almost USD 33.8 billion on ESKD and related disease, which corresponded to approximately $1 \%$ of the US government budget. Most of the expenditure is spent on outpatient and inpatient services, especially on cardiovascular and infection-related hospitalization. For dialysis-related expenditure, HD service is the most costly, with more than USD 25 billion expenditure in 2016. It is anticipated that the spending will continue to rise in the future. As a result, the USA signed an Executive Order on the Advancing American Kidney Health Initiative on 10 July 2019. There are mainly 3 goals under this initiative: (1) reduce the number of Americans developing ESKD by $25 \%$ by 2030, (2) aim for $80 \%$ of new American ESKD patients receiving home dialysis therapy or receiving kidney transplantation by 2025 , and (3) aim to double the number of kidneys available for transplantation by 2030 .

There are several possible ways to minimize dialysis expenditure. One effective way is to ensure a smooth transition from CKD to ESKD and avoid crash dialysis, which is associated with an extra cost of USD 18,500 per person [35]. This includes early recognition of high-risk patients, early planning of dialysis, early creation of dialysis access, and avoidance of emergency dialysis. Another way is to maximize the use of home dialysis therapy. Both USRDS and Canadian data demonstrated home dialysis treatment 
incurred a significant lower cost than incenter HD [26, 36]. By switching one patient from $\mathrm{HD}$ to $\mathrm{PD}$, the government can already save up to USD 20,000 per year per patient. Taking the total number of HD patients into account, the government would be able to save USD 300 million per year by just switching $30 \%$ of HD patients to $\mathrm{PD}$. Figure 3 shows the changes in the rate of patients undergoing home dialysis treatment in the USA from 1995 to 2016. It is noted that the rate dropped from 1995 to mid- and late-2010s and started to climb up again. This is in line with the Medicare Improvement for Patients and Providers Act passed in 2008 [37]. This prospective payment system introduced at that time for the ESKD program with the dialysis bundle included a new reimbursement strategy to incent US dialysis providers to place more patients on home dialysis. It placed most costs for dialysis care, especially the injected medications, within a bundle of services. It would be interesting to see how this trend will change under the new Advancing American Kidney Health Initiative in the USA introduced in 2019 [21]. Finally, maximization of transplantation by promotion of organ donation is also effective as the cost of maintaining a functioning graft is by far the cheapest and most cost-effective way to prolong survival in ESKD patients.

\section{Conclusion}

The burden of CKD on global healthcare system and economy from rising disease prevalence rate and induced demand on dialysis is in no doubt a huge issue. Although by far, there is no single solution that can be adopted universally to solve the problem, this roundtable discussion concluded that an integrated approach with early detection of CKD, prompt treatment to slow down progression, promotion of home-based dialysis therapy like PD and home HD, together with promotion of kidney transplantation is potentially an effective way to combat this ongoing worldwide challenge. The need to have primary, secondary, and tertiary prevention strategies is important [2]. However, local factors like cultural acceptance, patients' preference, and country's financial status should be considered before implementing any policy change.

\section{Statement of Ethics}

This is a conference report and a review, and no human or animal subject was involved. No statement of ethics is required.

\section{Conflict of Interest Statement}

P.K.T.L. received speaker honorarium from FibroGen and AstraZeneca. W.H.L. received speaker honorarium from Alexion and Astellas and an education grant from Novartis. B.W.T. received honoraria, consulting or speaker fees, or travel support from Böhringer Ingelheim, Novartis, Astellas, Sanofi, Servier, M.S.D., Astra-Zeneca, and Otsuka. X.Q.Y. received research study supports from Baxter Healthcare Corporation, Wanbang company, KyowaKirin, and AstraZeneca. G.S.K. and X.Q.Y. also acted as consultants for Fresenius Kabi, Baxter Healthcare Corporation, and AstraZeneca. Z.H.L. and J.C.H. are working as editor-in-chief and associate editor, respectively, for Kidney Diseases, and P.K.T.L., J.H.C., and X.Q.Y. are editorial board members for Kidney Diseases.

\section{Funding Sources}

This study and paper received no funding support.

\section{Author Contributions}

It is confirmed that all the authors contribute to the conception and design of the paper and have been involved in different sections of the paper on the acquisition, analysis, or interpretation of data for the paper. All the authors have involved in drafting the paper and revising it critically for important intellectual content and have approved the final version submitted to Kidney Diseases. All the authors agreed to be accountable for all the aspects of the paper in ensuring its accuracy or integrity.

\section{References}

1 International Society of Nephrology. 2019 United Nations high level meeting on UHC: moving together to build kidney health worldwide. 2019. Retrieved 20 July 2019 https://www.theisn.org/images/Advocacy_ 4_pager_2019_Final_WEB_pagebypage.pdf.

2 Li PK, Garcia-Garcia G, Lui SF, Andreoli S, Fung WW, Hradsky A, et al. Kidney health for everyone everywhere-from prevention to detection and equitable access to care. Kidney Int. $2020 \mathrm{Feb}$;97(2):226-32.
3 Foreman KJ, Marquez N, Dolgert A, Fukutaki K, Fullman N, McGaughey M, et al. Forecasting life expectancy, years of life lost, and allcause and cause-specific mortality for 250 causes of death: reference and alternative scenarios for 2016-40 for 195 countries and territories. Lancet. 2018;392(10159):2052-90.

4 Liyanage T, Ninomiya T, Jha V, Neal B, Patrice HM, Okpechi I, et al. Worldwide access to treatment for end-stage kidney disease: a systematic review. Lancet. 2015;385(9981): 1975-82.
5 Vanholder R, Annemans L, Brown E, Gansevoort R, Gout-Zwart JJ, Lameire N, et al. Reducing the costs of chronic kidney disease while delivering quality health care: a call to action. Nat Rev Nephrol. 2017;13(7):393409.

6 Lim WH, Johnson DW, McDonald SP, Hawley C, Clayton PA, Jose MD, et al. Impending challenges of the burden of end-stage kidney disease in Australia. Med J Aust. 2019 Oct; 211(8):374-e3. 
7 Kidney Health Australia 2016 report. https:// kidney.org.au/uploads/resources/kidneyhealth-australia-2016-annual-review.pdf.

8 Kazmi WH, Obrador GT, Khan SS, Pereira BJ, Kausz AT. Late nephrology referral and mortality among patients with end-stage renal disease: a propensity score analysis. Nephrol Dial Transplant. 2004;19(7):1808-14.

9 Australian and New Zealand Dialysis and Transplant Registry. 41st report. Chapter 1: incidence of end stage kidney disease. Adelaide: ANZDATA registry. 2018. https:// www.anzdata.org.au/wp-content/uploads/2018/11/c01_incidence_2017_ v1.0_20181121.pdf.

10 Canadian Organ Replacement Register. 2017. Accessed on 2020 Dec 20: https://www.cihi. $\mathrm{ca} /$ en/canadian-organ-replacement-registermetadata-corr.

11 Canadian Institute for Health Information. 2015. Accessed on 2020 Dec 20: https://www. cihi.ca/en/access-data-and-reports.

12 International Diabetes Federation. 2013. Accessed on 2020 Dec 20 https://idf.org/ e-library/epidemiology-research/diabetes-atlas.html.

13 Chinese National Renal Data System. Accessed on 2020 Dec 2 http://www.cnrds.net/ TxLogin.

14 Zhang L, Zhao MH, Zuo L, Wang Y, Yu F, Zhang H, et al. China Kidney Disease Network (CK-NET) 2015 annual data report. Kidney Int Suppl. 2019 Mar;9(1):e1-e81.

15 Yip WC, Hsiao WC, Chen W, Hu S, Ma J, Maynard A. Early appraisal of China's huge and complex health-care reforms. Lancet. 2012;379(9818):833-42.

16 National Health and Family Planning Commission of the People's Republic of China. China statistical year- book of health and family planning. Beijing, China: Peking Union Medical College Press; 2017.
17 Yao X, Chen S, Lei W, Shi N, Lin W, Du X, et al. The relationship between hemodialysis mortality and the Chinese medical insurance type. Ren Fail. 2019 Nov;41(1):778-85.

18 Tan X, Zhang Y, Shao H. Healthy China 2030, a breakthrough for improving health. Glob Health Promot. 2019;26(4):96-9.

19 USRDS. 2020. Accessed on 2020 Dec 20 https: //adr.usrds.org/2020/end-stage-renaldisease/11-international-comparisons.

20 Leung CB, Cheung WL, Li PK. Renal registry in Hong Kong-the first 20 years. Kidney Int Suppl. 2015;5(1):33-8.

21 Li PK, Rosenburg ME. Foreign perspective on achieving a successful peritoneal dialysis-first program. Kidney360. July 2020;1(7):680-4.

22 Wong CKH, Chen J, Fung SKS, Mok M, Cheng YL, Kong I, et al. Lifetime cost-effectiveness analysis of first-line dialysis modalities for patients with end-stage renal disease under peritoneal dialysis first policy. BMC Nephrol. 2020;21(1):42.

23 Choy AS, Li PK. Sustainability of the peritoneal dialysis-first policy in Hong Kong. Blood Purif. 2015;40(4):320-5.

$24 \mathrm{Li}$ PK, Szeto CC. Success of the peritoneal dialysis programme in Hong Kong. Nephrol Dial Transplant. 2008;23(5):1475-8.

25 Li PK, Law MC, Chow KM, Leung CB, Kwan $\mathrm{BC}$, Chung KY, et al. Good patient and technique survival in elderly patients on continuous ambulatory peritoneal dialysis. Perit Dial Int. 2007;27(Suppl 2):S196-201.

26 USRDS. 2018. Accessed on 2020 Dec 20: https://usrds.org/media/1738/v2_c11_intcomp_18_usrds.pdf.

27 Singapore renal registry annual report 2018. Accessed on 2020 Dec 3. https://www.nrdo. gov.sg/publications/kidney-failure.

28 HALT CKD programme. https://www.moh. gov.sg/news-highlights/details/speech-bymr-chan-heng-kee-permanent-secretaryministry-of-health-at-the-3rd-kidney-careconference-2019-25-may-2019.
29 Teo BW, Ma V, Xu H, Li J, Lee EJ; Nephrology Clinical Research Group. Profile of hospitalisation and death in the first year after diagnosis of end-stage renal disease in a multi-ethnic Asian population. Ann Acad Med Singap. 2010 Feb;39(2):79-87.

30 Leo CCH, Chan GC. Global perspectives in dialysis: Singapore. Kidney360. 2020;1(11): 1306-9.

31 Healthcare Schemes \& Subsidies, Singapore. Accessed on Dec 20, 2020: https://www.moh. gov.sg/cost-financing/healthcare-schemessubsidies.

32 Lin MY, Cheng LJ, Chiu YW, Hsieh HM, Wu $\mathrm{PH}$, Lin YT, et al. Effect of national pre-ESRD care program on expenditures and mortality in incident dialysis patients: A populationbased study. PLoS One. 2018;13(6):e0198387.

33 Harnett $\mathrm{P}$, Jones $\mathrm{M}$, Almond M, Ballasubramaniam G, Kunnath V. A virtual clinic to improve long-term outcomes in chronic kidney disease. Clin Med. 2018;18(5):356-63.

34 Major RW, Brown C, Shepherd D, Rogers S, Pickering W, Warwick GL, et al. The primarysecondary care partnership to improve outcomes in chronic kidney disease (PSP-CKD) study: a cluster randomized trial in primary care. J Am Soc Nephrol. 2019;30(7):1261-70.

35 Piwko C, Vicente C, Marra L, Jivraj F, Appel E, Wolpin J, et al. The STARRT trial: a cost comparison of optimal vs sub-optimal initiation of dialysis in Canada. J Med Econ. 2012; 15(1):96-104.

36 Beaudry A, Ferguson TW, Rigatto C, Tangri N, Dumanski S, Komenda P. Cost of dialysis therapy by modality in Manitoba. Clin J Am Soc Nephrol. 2018 Aug 7;13(8):1197-203.

37 Golper TA. The possible impact of the us prospective payment system ("bundle") on the growth of peritoneal dialysis. Perit Dial Int. 2013 Nov-Dec;33(6):596-9. 